\title{
EDITORIAL
}

\section{Neuroimaging in neuropsychiatric disorders}

I do not distinguish by the eye, but by the mind, which is the proper judge.

\section{Seneca $(5$ BC-65 AD)}

Where am ' $\mathrm{I}$ ' in the brain? This is not a new question, and by examining the structure of the brain and determining its interconnections, it is not unreasonable to assume that the self and the other functions of the brain can be found a home. Indeed, early in the 20th century, many scientists attempted to chart the workings of the mind onto the brain by detailing its anatomy and physiology. The task of localizing function, however, has proven to be much more difficult than initially presumed, with many regions of the brain subserving a variety of processes and having only loose coupling of structure and function. Hence, the majority of neuropsychological tests and the various brain capacities they tap lack regional specificity. This is a complex and sophisticated problem in the healthy brain that is compounded significantly by damage or disease. However, in the past two decades, neuroimaging has rekindled and renewed enthusiasm for unraveling brain function.

Amongst the many techniques and technologies that have been developed, functional magnetic resonance imaging (fMRI) has been one of the most exciting and perhaps the most widely used. It has permitted unprecedented access to the living brain and united scientists from a variety of scientific fields. In this special issue of Acta Neuropsychiatrica, we have brought together researchers with differing expertise and interests to showcase the range of applications of functional neuroimaging in humans.

Professor Veena Kumari (1) begins the discussion by synthesizing the imaging literature that has identified neural correlates of psychotherapeutic effects and points to the potential neurobiological substrates of various mental phenomena. This theme is revisited by Professor Michael Berk's group (2), in their description of links between depression and pain and the potential use of neuroimaging to explicate this association. Drs Morgan Haldane and Sophia Frangou (3) review the findings of neuroimaging studies in mood disorders suggesting that despite the use of disparate functional imaging modalities, the results show reassuring consistency. Similarly but more specifically, Drs Tracy and Sukhi Shergill (4) interrogate the neuroimaging evidence amassed from studies examining auditory hallucinations and suggest potential implications for future treatment and further research.

The remaining articles report findings from studies that have used a variety of imaging techniques and innovations. For instance, Erik Berntsen and colleagues (5) describe the development of a new stimulus for use within fMRI to lateralize language dominance, and Professor Terence Ketter's group (6) describes the findings from a spectroscopy study in bipolar disorder, noting a possible increase in occipital GABA concentrations. Finally, Dr Jim Lagopoulos and colleagues (7) detail new applications such as coupling fMRI with physiological measures and the use of cerebrovascular autoregulation as a neuroimaging tool (8), making the point that although neuroimaging has advanced considerably, there remains significant potential for further development.

As guest editors, we thank the editor Michael Maes for supporting this exciting issue and the many contributors for sharing their ideas and research. It is our hope that the articles in this issue are thought provoking and provide impetus for further research, and that in the near future, with further technological leaps, in addition to seeing with our minds eye, we will be able to see our minds with our eyes.

Gin S. Malhi, Jim Lagopoulos Neuroscience Research Group

Mayne Clinical Research Imaging Center School of Psychiatry University of NSW Sydney, Australia

\section{References}

1. Kumari V. Do psychotherapies produce neurobiological effects? Acta Neuropsychiatrica 2006;18:61-70. 
2. Williams LJ, Jacka FN, Pasco JA, Dodd S, Berk M. Depression and pain: an overview. Acta Neuropsychiatrica 2006;18:79-87.

3. Haldane M, Frangou S. Functional neuroimaging studies in mood disorders. Acta Neuropsychiatrica 2006; 18:88-99.

4. Tracy DK, Shergill SS. Imaging auditory hallucinations in schizophrenia. Acta Neuropsychiatrica 2006;18:71-78.

5. Berntsen EM, Rasmussen I, Samuelsen P et al. Putting the brain in Jeopardy: a novel comprehensive and expressive language task? Acta Neuropsychiatrica 2006; 18:115-119.
6. Wang PW, Sailasuta N, Chandler RA, Ketter TA. Magnetic resonance spectroscopic measurement of cerebral gamma-aminobutyric acid concentrations in patients with bipolar disorders. Acta Neuropsychiatrica 2006;18:120-126.

7. Lagopoulos J, Gordon E, Ward P. Differential bold responses to auditory target stimuli associated with a skin conductance response. Acta Neuropsychiatrica 2006; 18:105-114.

8. Lagopoulos J, Malhi GS, Ivanovski B et al. Cerebrovascular autoregulation as a neuroimaging tool. Acta Neuropsychiatrica 2006;18:100-104. 